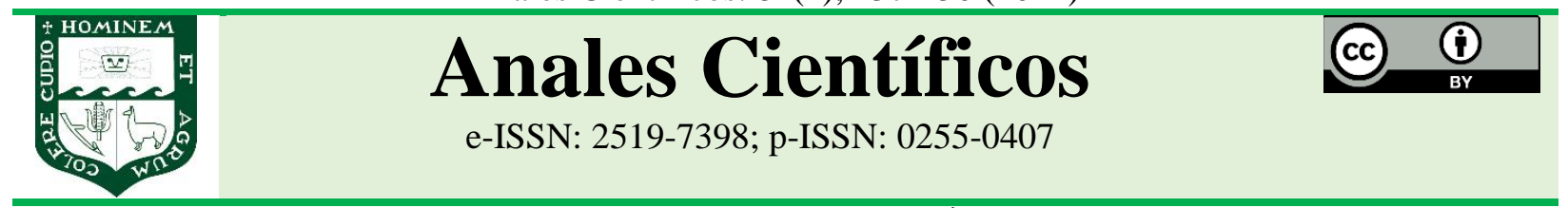

ARTÍCULO ORIGINAL - RESEARCH ARTICLE http://dx.doi.org/10.21704/ac.v82i2.1786

\title{
EFECTOS DE LOS REGÍMENES DE RIEGO SOBRE EL RENDIMIENTO Y EL USO DEL AGUA DEL CALABACÍN (Cucurbita pepo L.) EN CONDICIONES DE CAMPO
}

\section{Effects of irrigation regimes on the yield and water use of squash (Cucurbita pepo $L$.) under field conditions}

\author{
José Alexander Gil-Marín $^{1 *(D)}$; María Ximena Cordova-Rodriguez ${ }^{2}$ iD $;$ Nelson Montaño-Mata ${ }^{1}$ \\ ${ }^{1}$ Departamento de Ingeniería Agrícola, Núcleo Monagas, Universidad de Oriente, Estado Monagas, Venezuela. \\ ${ }^{2}$ Facultad de la Salud Humana, Universidad Nacional de Loja, Ecuador. \\ *E-mail: jalexgil2005@hotmail.com
}

Recibido: 18/02/2021; Aceptado: 08/12/2021; Publicado: 31/12/2021

\begin{abstract}
This work was carried out in the San Vicente sector, Maturín Municipality, Monagas State, Venezuela between the months of june-september 2015, with the objective of determining the effect of four irrigation deeps on the yield, production, quality of the fruits and efficiency in the use of water in the Shiraz cultivar of squash (Cucurbita pepo $L$ ). An experimental design of random blocks with three repetitions was used, with an arrangement of divided plots, where the main plot was the different irrigation deeps. The main plot had dimensions of $10 \mathrm{~m}$ long by $3 \mathrm{~m}$ wide, in the same four irrigation lines separated by $1.0 \mathrm{~m}$ were installed. Four irrigation criteria were applied, consisting of replacing the equivalent of $60 \%, 80 \%, 100 \%$ and $120 \%$ of the crop evapotranspiration (ETc). The parameters measured were the productive components and plant yield (t/ha). A type "A" evaporimeter tank was used to estimate the evapotranspiration of the squash. The $120 \%$ ETc treatment allowed the maximum yield and quality (in terms of fruits / plant, length, diameter and weight of the fruit), and the highest WUE. The maximum yield obtained was 33, 39 ton / ha. All the production functions of the yield and the quality parameters of the fruit perform a mathematical equation of the linear type with a high value of the coefficient of determination $\mathrm{R}^{2}$ above $90 \%$. The response factor of the squash crop yield $(2,35)$; greater than 1 indicates that the squash is very susceptible to water deficit.
\end{abstract}

Keywords. Water use efficiency | yield | drip irrigation | fruit quality

\section{RESUMEN}

El presente trabajo se realizó en el sector San Vicente, Municipio Maturín Estado Monagas, Venezuela entre los meses de junio-septiembre del 2015, con el objetivo de determinar el efecto de cuatro láminas de riego sobre el rendimiento, producción, la calidad de los frutos y eficiencia en el uso del agua en el cultivar Shiraz de calabacín (Cucurbita pepo L.). Se utilizó un diseño experimental de bloques al azar con tres repeticiones, con arreglo de parcelas divididas, donde la parcela principal fueron las diferentes láminas de riego. La parcela principal tuvo dimensiones de $10 \mathrm{~m}$ de largo por $3 \mathrm{~m}$ de ancho, en la misma se instalaron 4 líneas regantes separadas a $1 \mathrm{~m}$. Se aplicaron cuatro criterios de riego consistentes en reponer el equivalente al $60 \%, 80 \%, 100 \%$ y $120 \%$ de la 
evapotranspiración del cultivo (ETc). Los parámetros medidos fueron los componentes productivos y rendimiento planta (t/ha). Un tanque evaporímetro tipo "A" se utilizó para estimar la evapotranspiración del calabacín. El tratamiento $120 \%$ ETc permitió el máximo rendimiento y calidad (en términos de frutos/planta, longitud, diámetro y peso de la fruta), y la mayor EUA. El máximo rendimiento obtenido fue de 33,39 t/ha. Todas las funciones de producción del rendimiento y de los parámetros de calidad del fruto obedecen a una ecuación matemática del tipo lineal con un alto valor de coeficiente de determinación $\mathrm{R}^{2}$ por encima del $90 \%$. El factor de respuesta del rendimiento del cultivo de calabacín $(2,35)$; mayor de 1 indica que este cultivo es muy susceptible al déficit de agua.

Palabras clave: Eficiencia del uso del agua | rendimiento | riego por goteo $\mid$ calidad del fruto

Forma de citar el artículo (Formato APA):

Gil-Marín, J.A., Cordova-Rodriguez, M.X., \& Montaño-Mata, N. (2021). Efectos de los regímenes de riego sobre el rendimiento y el uso del agua del calabacín (Cucurbita pepo L.) en condiciones de campo. Anales Científicos. 81(2), 237-250. http://dx.doi.org/10.21704/ac.v82i2.1786

Autor de correspondencia (*): José A. Gil-Marín. Email: jalexgil2005@ hotmail.com

(C) Los autores. Publicado por la Universidad Nacional Agraria La Molina.

This is an open access article under the CC BY

\section{INTRODUCCIÓN}

Los frutos de calabacita o calabacín (Cucurbita pepo L.) se consumen principalmente inmaduros, como fruto verdura, tanto en el mercado nacional como en el de exportación. La calabacita es rica en carbohidratos y amino ácidos así como también contienen muchos minerales beneficiosos para los humanos. El calabacín ha sido considerado en el país como una hortaliza de segunda importancia, habiéndose desarrollado poca labor de investigación sobre el cultivo. Sin embargo, el aumento lento, pero significativo tanto en el área sembrada como en el consumo de este fruto están haciendo cambiar esta tendencia, notándose un creciente interés por el cultivo, tanto para el consumo local como para exportación.

En 2018 se cultivaron 6584 ha en Venezuela, con un rendimiento medio de $14,8 \mathrm{t} \mathrm{ha}^{-1}$, inferior al de Indonesia $\left(68,3 \mathrm{t} \mathrm{ha}^{-1}\right)$, Holanda $\left(66,73 \mathrm{t} \mathrm{ha}^{-1}\right)$ y España $\left(48,31 \mathrm{t} \mathrm{ha}^{-1}\right), \mathrm{y}$ apenas superior al promedio mundial $\left(13,53 \mathrm{t} \mathrm{ha}^{-1}\right)$ (FAO, 2020), lo que evidencia la necesidad de elevar el rendimiento nacional. Este crecimiento ha incentivado a que se realicen nuevos estudios para el desarrollo de tecnologías apropiadas a dicho cultivo y que el rendimiento pueda incrementarse, así como la utilidad económica. Dentro de este paquete tecnológico se encuentra el uso de los sistemas de riego por goteo del tipo superficial con diferentes estrategias de riego, como medio para aumentar el rendimiento y calidad de los frutos. Por otro lado, en los últimos años, la cantidad disponible de agua para la agricultura está disminuyendo en todo el mundo debido al rápido crecimiento de la población y la mayor incidencia de la sequía causada por el cambio climático y las diferentes actividades humanas (El-Mageed y Semida, 2015). Muchas de las zonas donde se cultiva calabacín ya están sufriendo modificaciones dramáticas en el entorno agrícola actual, que se agravará en el futuro, debido al cambio climático en curso (Anwar et al., 2013).

El cambio climático global y la creciente competencia por el agua dulce pueden tener graves impactos en los recursos hídricos y en el sector agrícola. El acceso al agua de riego es clave para reducir los impactos de la variabilidad y el cambio climático en la seguridad alimentaria. El riego es una práctica cada vez más importante para la agricultura sostenible en las regiones áridas y semiáridas (Fernández et al., 2020). Para resolver el problema de la escasez de agua en la agricultura, es necesario desarrollar tecnologías de gestión de ahorro de agua. Un manejo exitoso de la cantidad limitada de agua disponible para usos agrícolas depende de mejores prácticas agrícolas y una mejor comprensión de la productividad del agua (Howell, 2001; Jones, 2004). La técnica del Riego deficitario (es decir, riego por debajo del nivel óptimo de agua de cultivo) es una estrategia para el ahorro de agua mediante el cual los cultivos están sujetos a un 
cierto nivel de estrés hídrico durante un período particular o durante toda la temporada de crecimiento (Pereira et al., 2002).

El objetivo principal de usar el riego deficitario (RD) es aumentar el valor de la eficiencia del uso del agua (EUA) reduciendo la cantidad de agua aplicada con riego o reduciendo el número de eventos de riego (Kirda, 2002). Los efectos del RD sobre el crecimiento y la productividad de muchos vegetales y cultivos de campos han sido ampliamente investigados (Karam et al., 2006; Fereres y Soriano, 2007; Igbadun et al., 2008; Amer, 2011). Las evidencias de investigación han demostrado que RD tiene éxito en aumentar la productividad del agua para diferentes cultivos sin causar graves reducciones del rendimiento (Geerts y Raes, 2009). El-Dewiny (2011) señala disminuciones del rendimiento de calabaza de verano al aumentar los déficits de agua. Rouphael y Colla (2005) observaron que el rendimiento total y comercializable, el peso y el número de fruta se vieron afectados significativamente por la temporada de crecimiento y el sistema de riego y no por su interacción. El menor rendimiento registrado durante temporada de crecimiento de verano-otoño se relacionó con una reducción en el peso medio de la fruta y número de fruta.

Ertek et al. (2004) encontró en la época de verano el más alto el rendimiento de calabaza, con un tratamiento de riego con un coeficiente de planta-tina tipo A de 0,85 en Van, Turquía. Del mismo modo, el rendimiento de la fruta calabaza se vio significativamente afectado al aumentar las cantidades de riego (Al-Omran et al., 2005). Además, los valores EUA fueron generalmente aumentando con la cantidad de riego, pero disminuyó a un nivel de riego más alto. Este mismo autor señala que la eficiencia del uso del agua aumentó linealmente a medida que el agua de riego se incrementó debido al déficit en el nivel de riego y disminuido por nivel de riego excesivo. Sin embargo, los efectos de RD son específicos de los cultivos.

Por lo tanto, es necesario evaluar el impacto de las estrategias de RD en campos abiertos con varios años de experimentación, antes de generalizar la estrategia de riego más apropiada para el método de programación que será adaptado en una ubicación específica para un determinado cultivo (Scholberg et al., 2000). Aunque hay muchas investigaciones sobre las influencias de las limitaciones regulada del agua en los parámetros de rendimiento y calidad, relativamente hay pocos trabajos relacionados con el riego de calabacín en nuestras condiciones locales del oriente del país y Maturín. El objetivo de la investigación fue determinar las influencias de diferentes niveles de riego sobre el rendimiento, calidad del fruto, producción y eficiencia en el uso del agua (EUA) en el cultivo de calabacín.

\section{MATERIALES Y MÉTODOS}

El trabajo se realizó en el sector de San Vicente, municipio Maturín, estado Monagas, Venezuela; entre los meses de marzo y septiembre del 2015. Su ubicación geográfica está comprendida entre los $9^{\circ} 44^{\prime} 37^{\prime \prime}$ de latitud Norte y los $63^{\circ} 15^{\prime} 59^{\prime \prime}$ de longitud Oeste, a una altitud de 51 m.s.n.m (Figura 1). El clima de la zona según Holdridge citado por Montaño et al. (2018), es del tipo Bosque Seco Tropical, caracterizado por presentar una estación lluviosa de mayo a diciembre y una estación seca de enero a abril, con una precipitación media anual de 1219,6 mm, una temperatura media anual de $25,9^{\circ} \mathrm{C}$, con una evapotranspiración potencial de $1,372 \mathrm{~mm}$ y una evaporación de 1,573 mm al año.

El suelo predominante es de textura franco arenosa y se clasifican taxonómicamente como ultisol (paleustults), que se caracteriza por ser muy lixiviado, con $\mathrm{pH}$ de 4,7; baja capacidad de intercambio catiónico y bajo porcentaje de materia orgánica (Gil et al., 2014) (Tabla 1)

Se utilizó el cultivar de calabacín Shiraz a un espaciamiento de $0,3 \mathrm{~m}$. El experimento se presentó en un diseño de parcela dividida con tres repeticiones (Figura 2) y ocho tratamientos producto de cuatro niveles del factor riego como parcela principal $(60,80$, 100 y $120 \%$ ETc) y dos niveles de la edad de trasplante como sub-parcela (14 y 21 días). La programación del riego se realizó en días alternos sobre la base de la evapotranspiración del cultivo de referencia (ETo) y los coeficientes del cultivo $(\mathrm{Kc})$. $\mathrm{La}$ evapotranspiración del cultivo de referencia (ETo) se estimó utilizando el Método de la Tina de Evaporación Tipo A de la FAO. 
La lectura diaria de evaporación registrada en la tina clase A se convirtió a ET de referencia (ETo) al multiplicar por el coeficiente de la tina $(\mathrm{Kp})$ de $(0,80)$; valor obtenido después de considerar la humedad relativa y la velocidad del viento predominante de la zona. Por último, la ET del cultivo (ETc) se obtuvo multiplicando ETo por el coeficiente del cultivo (Kc): $\mathrm{ETc}=\mathrm{ETo} \mathrm{x} \mathrm{Kc}$, como lo recomienda Allen et al. (1998).

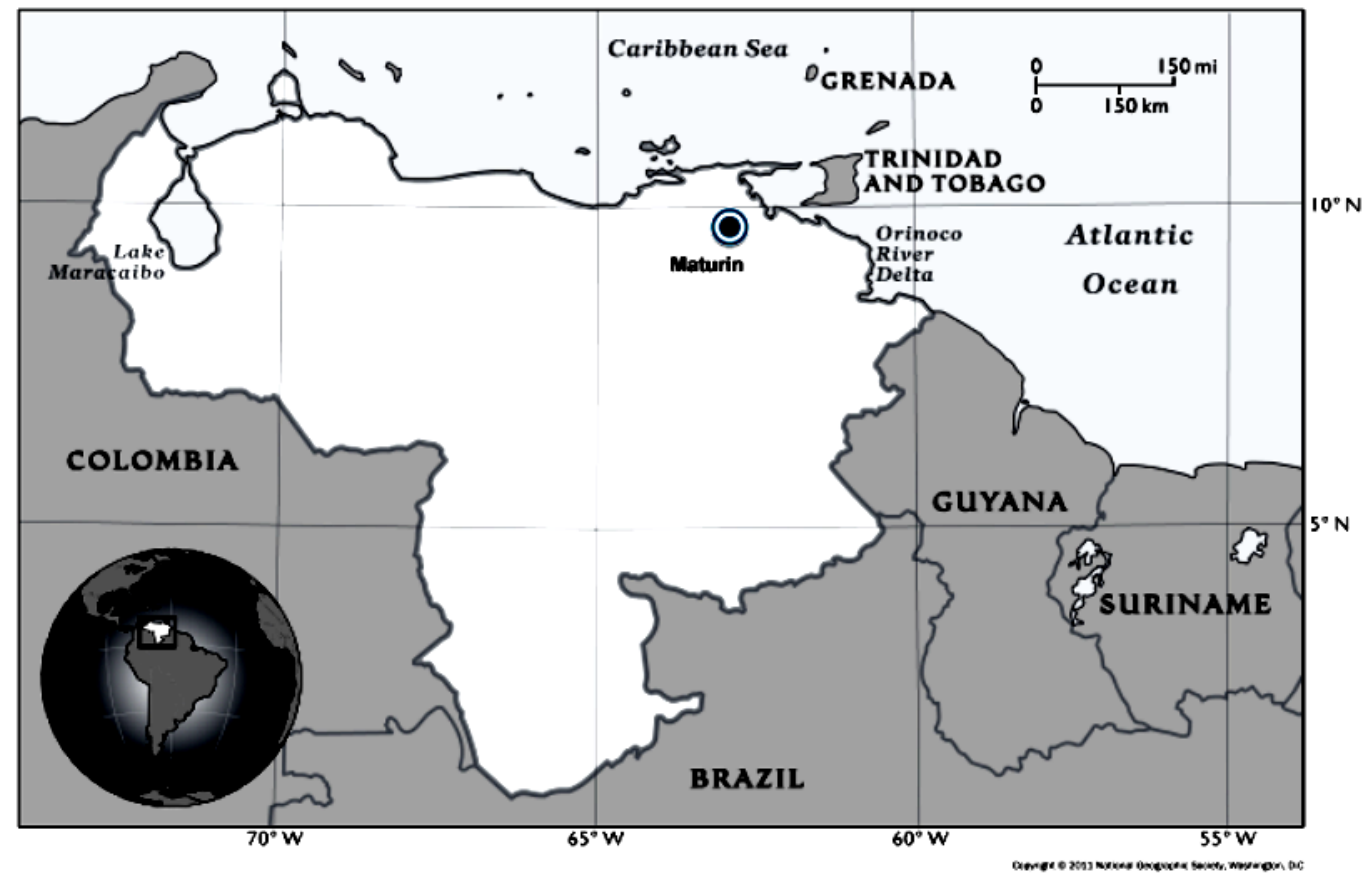

Figura 1. Ubicación geográfica de la Ciudad de Maturín. Estado Monagas. Venezuela. Fuente: (Gil, et al., 2012).

Tabla 1. Algunas propiedades físicas y químicas del suelo donde se desarrolló el trabajo.

\begin{tabular}{lcl}
\hline Características & Valor & Método \\
\hline Textura & Fa & Bouyoucos \\
pH & 4,7 & Potenciómetro \\
Materia Orgánica (\%) & 3,37 & Walkley y Blank \\
CE (micro S/m) & 192,4 & Conductimetro
\end{tabular}

$\overline{\text { Análisis realizado en el laboratorio de suelos de la Universidad de }}$ Oriente, Núcleo Monagas, Campus los Guaritos. (Gil et al.2014).

Los valores de Kc locales estimados para riego por goteo y calabacín en este estudio fueron 0,$3 ; 0,6 ; 0,90$ y 0,75 para las diferentes etapas del cultivo. Cada etapa duro aproximadamente 25 días. Las parcelas estuvieron constituidas por cuatro laterales de $10 \mathrm{~m}$ de longitud, separadas a $1 \mathrm{~m}$ entre ellos, con goteros de riego cada $0,33 \mathrm{~m}$, considerándose para efectos de evaluación solo las dos hileras centrales. Se instaló un sistema de riego por goteo con goteros integrados tipo Flat dripper line marca Aquadrop, con un espesor de pared de $0,3 \mathrm{~mm}$ y un caudal de $1,0 \mathrm{~L} / \mathrm{h}$. El área total del experimento fue de $448 \mathrm{~m}^{2}$, teniendo las parcelas principales un área de $30 \mathrm{~m}^{2}$ y las sub-parcelas de $5 \mathrm{~m}^{2}$. La preparación del terreno, se realizó con tres pases de rastra, con el objetivo de eliminar las malezas presentes y dar al suelo una soltura que permitiera el desarrollo del sistema radical del cultivo y se surco el terreno, con separación de 1,00 m.

Las plántulas fueron obtenidas en bandejas de germinación de 162 alvéolos, y el sustrato utilizado fue una mezcla de turba y sustrato de corteza de pino (PROFORCA), en una proporción de 3:1, realizándose la siembra de la primera edad (14 días después de la germinación, ddg y 18 días después de la siembra, dds), el día 15 de abril de 2015 y la segunda edad (21 días después de la germinación, ddg y 25 días después de la siembra, dds) el 22 de abril de 2015. La cantidad de semillas utilizada fue de 1 por alvéolo para un total 
máximo de 162 semillas por bandeja y 2916 por todo el ensayo. La germinación se inició a los 4 días luego de la siembra. La frecuencia de riego de las plántulas fue de dos veces al día, aplicándose una en la mañana y la otra en la tarde. El control de malezas, cuando la incidencia de estas lo requirió, se efectuó de forma manual o con herbicida. Asimismo, se realizó apropiado control fitosanitario del ensayo.

La fertilización se realizó a través del sistema de riego usando diariamente fertilizantes solubles de fórmula
27,5-49-0 durante los primeros 30 días y la fórmula 2814-30 durante el resto de la etapa del cultivo. La cantidad de fertilizante aplicado estuvo en el rango de 200-500 g.dia ${ }^{-1}$. Se hicieron los ajustes necesarios en el sistema con el fin de mantener iguales las dosis aplicadas por planta, independientemente de los diferentes volúmenes de empleados en cada tratamiento de riego.

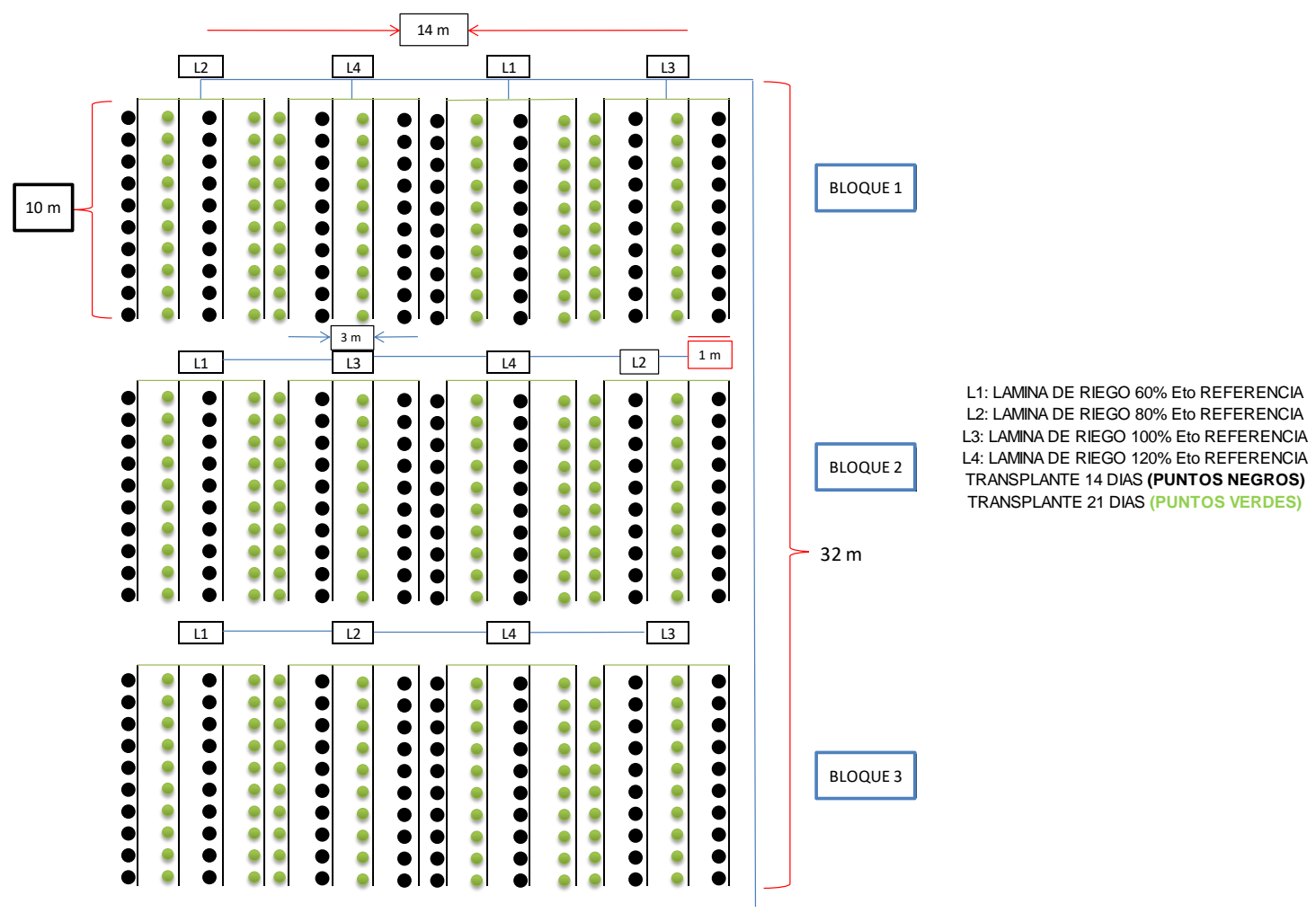

Figura 2. Plano experimental y distribución de los tratamientos con el cv. Shiraz (Cucurbita pepo L.) dos edades de trasplante y cuatro estrategias de riego en el sistema de riego Perú-San Vicente, Municipio Maturín, estado Monagas, Venezuela.

En nuestro estudio, las relaciones entre rendimiento y ET se determinaron por el modelo de Stewart (Doorenbos y Kassam, 1979).

$$
(1-\mathrm{Ya} / \mathrm{Ym})=\mathrm{k} \text { y }(1-\mathrm{ETa} / \mathrm{ETm})
$$

donde Ym y Ya son los rendimientos máximo y real, ETx y ETa son la evapotranspiración máxima y real, y Ky es el factor de respuesta del rendimiento que representa el efecto de una disminución de la evapotranspiración sobre las pérdidas de rendimiento. La ecuación 1 es una función de productividad del agua y puede ser aplicada a todos los cultivos agrícolas; es decir, herbáceos, arbóreos y vides.

Mientras la eficiencia del uso del agua de riego (EUA) se estimó con la ecuación (Howell et al., 1990):

EUA $\left(\mathrm{kg} \mathrm{m}^{-3}\right)=$ Yi/ETa... 
Donde Yi es el valor de rendimiento de cada parcela de tratamiento $\left(\mathrm{kg} \mathrm{ha}^{-1}\right)$.

Los calabacines de cada parcela unitaria fueron cosechados y a los frutos se le determino las variables longitud del fruto $(\mathrm{cm})$, diámetro del fruto $(\mathrm{cm})$, número de frutos y peso de frutos frescos. Los análisis estadísticos fueron realizados utilizando el Proc GLM (modelos lineales generales) procedimiento de SAS (SAS Institute, Inc., Cary, N. C.) en un nivel de significancia de $p \leq 0,05$. El análisis de varianza (ANOVA) se realizó para determinar las diferencias entre tratamientos para cada parámetro como aplicable al diseño completo de bloques al azar dispuestos en procedimiento de parcelas divididas. Las medias de tratamiento se compararon con el Procedimiento de Duncan a 0,05 nivel de probabilidad.

\section{RESULTADOS Y DISCUSIÓN}

Estos estudios se realizaron para determinar si las respuestas del rendimiento y sus componentes a los regímenes con riego completo y varios déficits pueden utilizarse en la planificación del riego. Los resultados indican que los niveles de riego fueron significativos sobre todas estas variables.

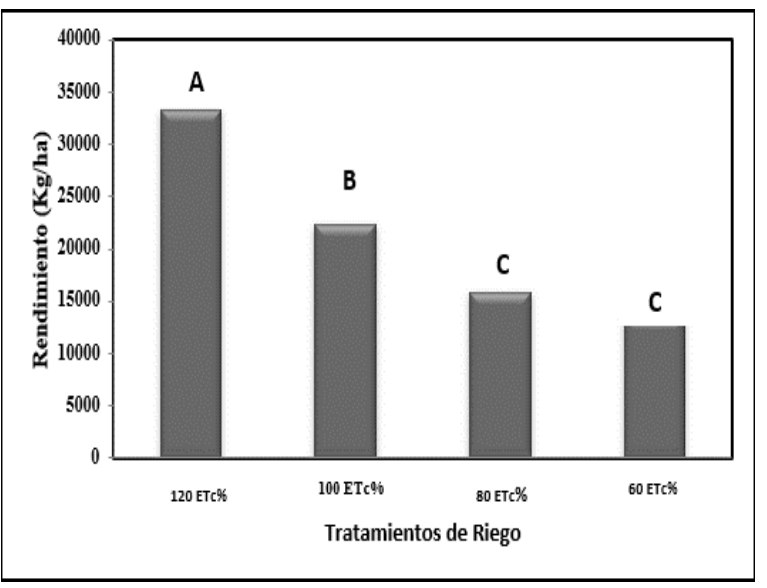

Figura 3. Efectos de los diferentes tratamientos o estrategias de riego sobre el rendimiento del fruto del cultivar cv. Shiraz de calabacín (Cucurbita pepo L.). Prueba de Duncan al 5\% de probabilidad. Letras iguales indican promedios estadísticamente iguales.

\section{Rendimiento $\left(\mathbf{t ~ h a}^{-1}\right)$}

El efecto de los niveles de riego en el rendimiento de los frutos, monitoreado durante la temporada para cada tratamiento se presenta en la Figura 3. Los rendimientos fueron afectados de forma altamente significativa $(p \leq 0,01)$, por los niveles de riego. La prueba de rango múltiples de Duncan al $5 \%$ de probabilidad, reflejó que la dosis de riego que arrojó el mayor rendimiento fue la lámina $120 \%$ ETc con una media de 33,39 $\mathrm{t} \mathrm{ha}^{-1}$, y el de menor rendimiento lo obtuvo el tratamiento $60 \%$ ETc con 13,57 $\mathrm{t} \mathrm{ha}^{-1}$. El cambio de la dosis de riego de $120 \%$ a $60 \%$ ETc, disminuyo en un 59,36\% el rendimiento de frutos, lo que evidencia el efecto negativo del estrés hídrico en este cultivo. Esto posiblemente se debe al hecho de que el agua aplicada con $120 \%$ ETc cumple de manera adecuada con los requerimientos hídricos del cultivo para la zona de estudio. Estos resultados indican que el incremento de las cantidades de riego tiene un efecto significativo y positivo en el rendimiento y los componentes del rendimiento del calabacín. Resultados similares también se reportan en otros estudios. Amer (2011) encontró que el rendimiento, el peso, el número, el diámetro y la longitud de la fruta del calabacín disminuyeron significativamente por la reducción del agua de riego aplicada por debajo del $100 \%$ de ETc. Erteket et al. (2004) señala que las cantidades de riego tuvieron efectos significativos sobre el rendimiento y sus componentes en calabaza de época de verano y las relaciones entre el rendimiento y sus componentes con la cantidad de agua de riego fueron de tendencia lineal y positiva. Al-Omran et al. (2005) determinó que el rendimiento de frutos de calabaza fue mayor para condiciones de riego sin estrés (100 y 120\% de la evapotranspiración estimada del cultivo) en comparación con las condiciones de riego estresadas (60 y 80\% de la evapotranspiración estimada del cultivo). El-Gindy et al. (2009) mostraron que una mayor cantidad de riego $(80 \%$ de ETc) proporcionó mayores rendimientos de peso fresco, diámetros de frutos, número de frutos por planta y longitudes de frutos en calabazas de verano regadas por goteo, en comparación con aquellas con una menor cantidad de riego (60\% de ETc) . 
Pero El-Dewiny (2011) encontró que la reducción de la cantidad de riego de 100 a $85 \%$ produjo un aumento en el rendimiento de la calabaza que se regó por goteo con tratamientos de 100, 85, 70 y 50\% de ETc. Sin embargo, el aumento de los déficits de agua redujo el rendimiento de la calabaza en la época de verano. Uno de los objetivos del riego deficitario es ahorrar agua reduciendo las tasas de aplicación por debajo del 100\% de la ETc, sin afectar negativamente el rendimiento de la fruta. Los períodos de crecimiento más sensibles del calabacín al estrés hídrico son la floración y la formación de frutos. Por lo tanto, la ocurrencia de cualquier estrés hídrico en estas etapas de crecimiento podría conducir al desarrollo de pudrición del extremo de la flor y frutos malformados.

La razón del mayor rendimiento con los tratamientos de riegos de 120 y $100 \%$ ETc, está en que las plantas pueden absorber agua más fácilmente sin experimentar ningún efecto de estrés en comparación con los tratamientos de menor cantidad de agua. Los resultados indicaron que se puede recomendar el tratamiento con riego completo $120 \%$ ETc con frecuencia de riego de 2 días. La respuesta fisiológica de la calabaza a diferentes niveles de estrés hídrico se puede utilizar para la optimización y sostenibilidad de la producción de cultivos en áreas donde las fuentes de agua son limitadas o caras. Passioura y Angus (2010) indican que las disminuciones en el crecimiento de las plantas en zonas de agricultura semiárida podrían ser el resultado de reducciones en la transferencia de asimilados de las hojas a los frutos debido a las condiciones de suministro de agua cada vez más desfavorables. Esta observación es aparentemente pertinente al calabacín producido en condiciones de estrés hídrico. Estos mismos autores también señalan que bajo estrés hídrico, los ciclos de vida de las plantas anuales se acortan y la senescencia de las hojas se acelera debido a la reducción de la absorción de agua por las raíces. Es importante resaltar que los rendimientos obtenidos están por encima de la media nacional, 14,8 $\mathrm{t} \mathrm{ha}^{-1}$ (FAO, 2020), lo que probablemente se deba a condiciones climáticas favorables, baja variación de temperatura y humedad, y al uso de tecnologías como riego por goteo y la técnica del riego deficitario. Los mayores rendimientos obtenidos en este experimento resultan superiores a los reportados por El-Mageed y Samida (2015), quienes obtuvieron rendimientos de $14 \mathrm{t}^{*} \mathrm{ha}^{-1}$, muy parecidos a los de Wetzel y Stone (2019) 33,9 $\mathrm{t}^{*} \mathrm{ha}^{-1}$ y Okasha et al. (2020) con valores de 28 a $22 \mathrm{t}^{*} \mathrm{ha}^{-1}$, pero inferiores a los reportados por Kusla et al. (2014) con $80 \mathrm{t}^{*} \mathrm{ha}^{-1} \mathrm{y}$ Rolbiecki et al. (2017) con $60 \mathrm{t}^{*} \mathrm{ha}^{-1}$.

\section{Agua aplicada y agua utilizada Eficiencia del Uso del Agua de Rendimiento}

En la Tabla 2 muestra la programación de riego real llevada a cabo durante el ensayo, expresada como porcentaje de satisfacción de la evapotranspiración de cultivo (ETc). También se presenta láminas totales de riego y precipitación efectiva aplicados en los diferentes tratamientos, así como los rendimientos y las Eficiencias del uso del agua (EUA) obtenidas. Se realizó un análisis de varianza del rendimiento y de la EUA para estudiar las diferencias producidas en los mismos, como consecuencias de los distintos tratamientos hídricos aplicados durante el ensayo. Tanto las producciones como los valores de EUA de rendimiento presentaron diferencias altamente significativas $(\mathrm{p}<1 \%)$.

Tabla 2. Tratamientos hídricos y láminas aplicadas a los diferentes tratamientos de riego y sus efectos sobre el rendimiento y eficiente uso del agua.

?

\begin{tabular}{cccccccc}
\hline & $\begin{array}{c}\text { Nivel de } \\
\text { Tratamiento }\end{array}$ & $\begin{array}{c}\text { Precipitación } \\
\text { Efectiva } \\
(\mathbf{\%})\end{array}$ & $\begin{array}{c}\text { Riego de } \\
\text { Asiento } \\
(\mathbf{m m})\end{array}$ & $\begin{array}{c}\text { Riego } \\
\text { Efectivo } \\
(\mathbf{m m})\end{array}$ & $\begin{array}{c}\text { Agua } \\
\text { Total } \\
(\mathbf{m m})\end{array}$ & Rendimiento & EUA \\
\hline $\mathrm{T}_{1}$ & 120 & 129,9 & 16,78 & 199,2 & 345,88 & 33385,00 & $\mathbf{K g} / \mathbf{m 3}$ \\
$\mathrm{T}_{2}$ & 100 & 129,9 & 16,78 & 166 & 312,68 & 22490,00 & $7,19 \mathrm{~b}$ \\
$\mathrm{~T}_{3}$ & 80 & 129,9 & 16,78 & 132,8 & 279,28 & 15834,00 & $5,67 \mathrm{c}$ \\
$\mathrm{T}_{4}$ & 60 & 129,9 & 16,78 & 99,6 & 246,28 & 13573,00 & $5,51 \mathrm{c}$ \\
\hline
\end{tabular}


Las EUA han oscilado entre $9,65 \mathrm{~kg} \mathrm{~m}^{-3}$ para el tratamiento más abundante $\mathrm{T}_{1} 120 \%$ ETc y $5,51 \mathrm{~kg} \mathrm{~m}^{-}$ ${ }_{3}^{3}$ para el tratamiento más restrictivo $\mathrm{T}_{4} 60 \%$ ETc. Los tratamientos $\mathrm{T}_{2}$ y $\mathrm{T}_{3}$ ocuparon ese mismo orden en la EUA de este cultivo. La mayor eficiencia del uso del agua correspondió al tratamiento $T_{1}$ con $9,65 \mathrm{~kg} \mathrm{~m}^{-3}$, seguido de $\mathrm{T}_{2}$ con $7,19 \mathrm{~kg} \mathrm{~m}^{-3}$. No hubo diferencia significativa entre los tratamientos $\mathrm{T}_{3} 80 \%$ ETc y $\mathrm{T}_{4}$ $60 \%$ ETc, siendo ambos inferiores a $\mathrm{T}_{1} \mathrm{y}_{2}$. Los resultados señalan una relación directamente proporcional entre la EUA y los niveles de ETc aplicados en los riegos.

Algunos trabajos sugieren que un valor alto de EUA debería estar asociado con rendimientos altos (o aceptables), particularmente en áreas con escasez de agua. Los resultados obtenidos en este trabajo son similares a los encontrados por Al-Omran et al. (2005), quienes indican que la eficiencia del uso del agua fue máxima con los tratamientos de mayor aplicación de agua (100 y 120\% de ETc) en comparación con aquellos de menor aplicación (60 y $80 \%$ de ETc). En forma general, de acuerdo con los resultados de diferentes estudios, los tratamientos con tasas más bajas de ETc producen valores de EUA más altos. Ertek et al. (2004) obtuvo los valores de EUA más altos para la calabaza de verano en las condiciones de riego más bajas (45\% de evaporación de bandeja de Clase A). El-Gindy et al. (2009) determinaron que los valores de EUA de calabazas en época de verano, regadas por goteo con menores cantidades de agua (60\% de ET c), eran superiores a los valores regadas con mayores cantidades de agua (80\% de ETc). El-Dewiny (2011) mostró que el valor de EUA más bajo se obtuvo para la calabaza con riego completo y que los valores de EUA aumentaron con el aumento del déficit de agua. La razón de una mayor EUA en cultivos bajo estrés hídrico se debe a que las plantas estresadas se marchitan mucho más que las plantas no estresadas y la marchitez ocurre invariablemente en momentos en que el déficit de presión de vapor de la atmósfera es grande (Bloch et al., 2006).

En las condiciones locales, Gil et al. (2012) en el cultivo de ají, reportan rangos de EUA de rendimiento entre 8,25 a $24,24 \mathrm{~kg} / \mathrm{mm}$, y la máxima EUA la alcanzo con el $80 \%$ Etc. Mientras que, en el cultivo de melón, Gil et al. (2014), observaron valores de EUA entre
57,20 y $64,860 \mathrm{~kg} / \mathrm{mm}$, y la máxima EUA también se alcanzó con el $80 \%$ de la ETc. Estos resultados nos indican que el cultivo de calabacín tiene una EUA de rendimiento mayor que los cultivos de ají y melón, ya que con cada milímetro de agua aplicado fue capaz de producir como mínimo $55 \mathrm{~kg}$ de fruta fresca de este cultivo. Los estudios encontrados sobre la utilización de la EUA para comparar diferentes especies cultivables, o para indicar la eficiencia fotosintética de distintos esquemas de cultivos a través de la EUA, y la opción de elegir métodos de riego en función de la EUA asientan la alternativa de utilizar a esta variable como un indicador universal que permita la elección de sistemas de producción sustentables. Se incluye al concepto de sustentabilidad dado que en el manejo eficiente del agua están reflejadas indirectamente las prácticas agrícolas que se realizan, en una sucesión de cultivos planteada, considerando la importancia de la incorporación de materia orgánica a los lotes, que aumentará la capacidad de captación y almacenaje de agua del suelo. A su vez tendrá influencia en la porosidad, generando micro canales de mayor tamaño capaces de retener agua útil, estos dos factores mencionados mejorarán la oferta hídrica, consecuentemente los cultivos tendrán una mayor disponibilidad para aprovechar el agua, reflejado en una mayor eficiencia del uso.

\section{Funciones de producción de rendimiento}

En este trabajo se estudiaron las diferentes funciones de producción (Solomon, 1985; Howell et al., 1990) que relacionan está con el volumen total de agua recibido por el cultivo. El método estadístico utilizado fue el de la regresión simple. Se utilizaron funciones polinómicas de primer y segundo grado, determinándose en cada caso sus coeficientes de determinación $\left(\mathrm{R}^{2}\right)$, el nivel de significación estadístico y el análisis residual, que incluye la varianza, la desviación típica y la media. Para el caso del rendimiento de calabacín la función matemática obtenida, fue una ecuación lineal del tipo $\mathrm{Y}=$ 199,03ETc - 37,599 con un coeficiente de determinación altamente significativo $(\mathrm{P}<1 \%$ ), cuyo valor de $\mathrm{R}^{2}=0,9221$, significa que aproximadamente el $92 \%$ de la variación en el rendimiento del calabacín esta explicado por la lámina de agua aplicada (Figura 4). Estos resultados nos indican el efecto directamente proporcional que existe entre el rendimiento y las 
láminas de riego, en donde cada incremento unitario del porcentaje de la lámina de riego es capaz de producir un incremento de más de $199 \mathrm{~kg}$ en el rendimiento del calabacín. El-Mageed y Semida (2015), trabajando con tres niveles de riego y el híbrido Hi Tech de calabacín en el valle del Cairo, Egipto, reporta en esta relación una ecuación de segundo grado del tipo $\mathrm{Y}=2 \mathrm{E}-06 \mathrm{ETc}^{2}+0,0185 \mathrm{Etc}-31,89$ con un coeficiente de determinación de $\mathrm{R}^{2}=1,0$.Las relaciones directamente proporcionales entre las láminas de riego aplicadas y los rendimientos han sido reportadas por Bekele y Tilahum (2007) en Etiopía e Igbadun et al. (2012) en Nigeria, lo que también concuerda con Ertek et al. (2006); Wang et al. (2009); He-xi et al. (2011), quienes señalan que los rendimientos de frutos de pepino aumentaron con el incremento de las láminas de riego aplicadas. Este resultado se puede derivar del hecho de que cuando hay suficiente agua disponible en el suelo se produce un aumento de la absorción de agua $\mathrm{y}$ nutrientes $\mathrm{y}$, en consecuencia, un aumento de los mecanismos metabólicos en las plantas que conducen a un mayor peso, longitud y número de frutos por planta. Por otro lado, el estrés por sequía también afecta la estabilidad de la membrana celular y las características de intercambio de gases en las plantas (Hamed, 1988), lo que puede cambiar la selectividad del agua y los nutrientes debido al cambio en el potencial osmótico de las plantas estresadas en comparación con las cultivadas con tratamientos de riego normales. En la Figura 4, se observa que las producciones máximas por encima de $33 \mathrm{t} \mathrm{ha}^{-1}$, se obtuvieron con el tratamiento $120 \%$ de la ETc $(345,88$ $\mathrm{mm}$ de agua), con diferencias estadísticas con respecto al resto de los otros tratamientos. Esto significa que para el cv. Shiraz de Calabacín en el Valle del Rio Guarapiche, durante la época seca, la dosis de riego aplicada debe ser de $120 \%$ de la ETc, en nuestro caso estuvo representada por una dosis diaria promedio máximo de $4 \mathrm{~mm} /$ día o 1,32 litros/día/planta, para las épocas de floración y formación del fruto.

Gil et al. (2014) para el cultivo de melón encontró unas tendencias muy diferentes, ya que las producciones máximas por encima de $29 \mathrm{t} \mathrm{ha}^{-1}$, se obtuvieron con el tratamiento $80 \%$ de la ETc (421 mm de agua), con diferencias estadísticas con respecto al tratamiento excedentario $(120 \%$ ETc).

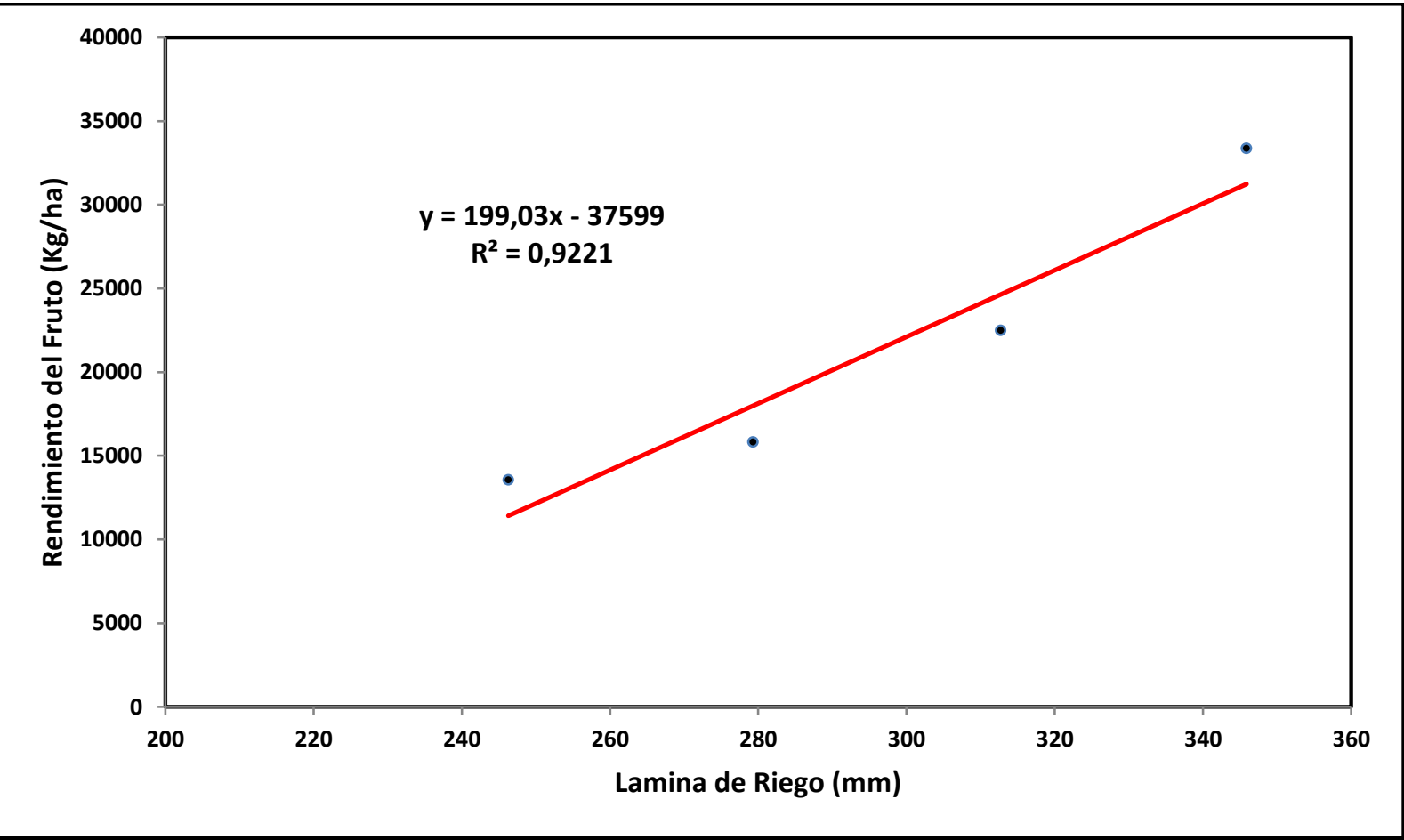

Figura 4. Función de producción que relaciona el rendimiento del cultivo de calabacín (Cucurbita pepo L.) cv. Shiraz y el agua total (riego + precipitación efectiva) recibida por el cultivo. 
En cambio en el calabacín para el factor riego, siempre se observó a lo largo de todas las cosechas, que el tratamiento superior fue el 1 (120\% de la ETc), lo que nos indica una tendencia directamente proporcional entre la lámina aplicada y la producción del cultivo, que pone en manifiesto las exigencias hídricas de este cultivo.

\section{Funciones de producción de los componentes de} calidad del fruto

Se determinó un alto nivel de relación lineal entre frutos/planta, longitud, peso y diámetro del fruto, con la cantidad de agua aplicada. Las ecuaciones lineales fueron las siguientes: Longitud de fruto $=0,0264 \mathrm{ETc}$ $+12,577$ con $R^{2}=0,9699$ (Figura. 5.a); Peso de fruto $=2,0353 \mathrm{ETc}-120,51$ con $\mathrm{R}^{2}=0,9777$ (Fig. 5. b.); diámetro de fruto $=0,0054 \mathrm{ETc}+3,5575$ con $\mathrm{R}^{2}=$ 0,9995 (Fig. 5. c.) y Frutos/plantas $=0,1579 E T c-$ $28,3772 \mathrm{R}^{2}=0,9894$ (Figura. 5.d). Los regímenes de riego tuvieron un efecto altamente significativo sobre los diferentes parámetros de calidad. Las variables más afectadas por las láminas fueron frutos/planta y numero de frutos. Estos resultados están en línea con los registrados por Adams (2002).

El mayor rendimiento registrado durante esta investigación de $33,385 \mathrm{tha}^{-1}$, con el tratamiento $120 \%$ ETc se debió a un aumento en el peso del fruto (595 g), $\mathrm{N}^{\circ}$ de frutos/plantas $(27,50)$, diámetro $(5,42 \mathrm{~cm})$ y longitud del fruto $(21,53 \mathrm{~cm})$ que los obtenidos en los otros tratamientos. Amer (2011) observo en calabacín, un mayor peso, número, diámetro y longitud de la fruta fresca con el tratamiento del 100\% ETc con riego por goteo. Díaz-Pérez y Eaton (2015) en berenjena reportan el efecto de disminución del régimen de riego sobre las variables número de frutos y peso del fruto y determinaron que el tratamiento más crítico fue el de 33\% ETc. Malash et al. (2005); Ozbahce y Tari (2010) en tomate y Wajid (1990) trabajando en maíz, también señalan que el estrés hídrico resultó en una reducción de la longitud de la fruta, el volumen de la fruta y diámetro del fruto. Nuestros resultados están en general de acuerdo con los hallazgos de las investigaciones antes mencionados sobre el efecto del estrés en los parámetros de calidad de la fruta del calabacín.
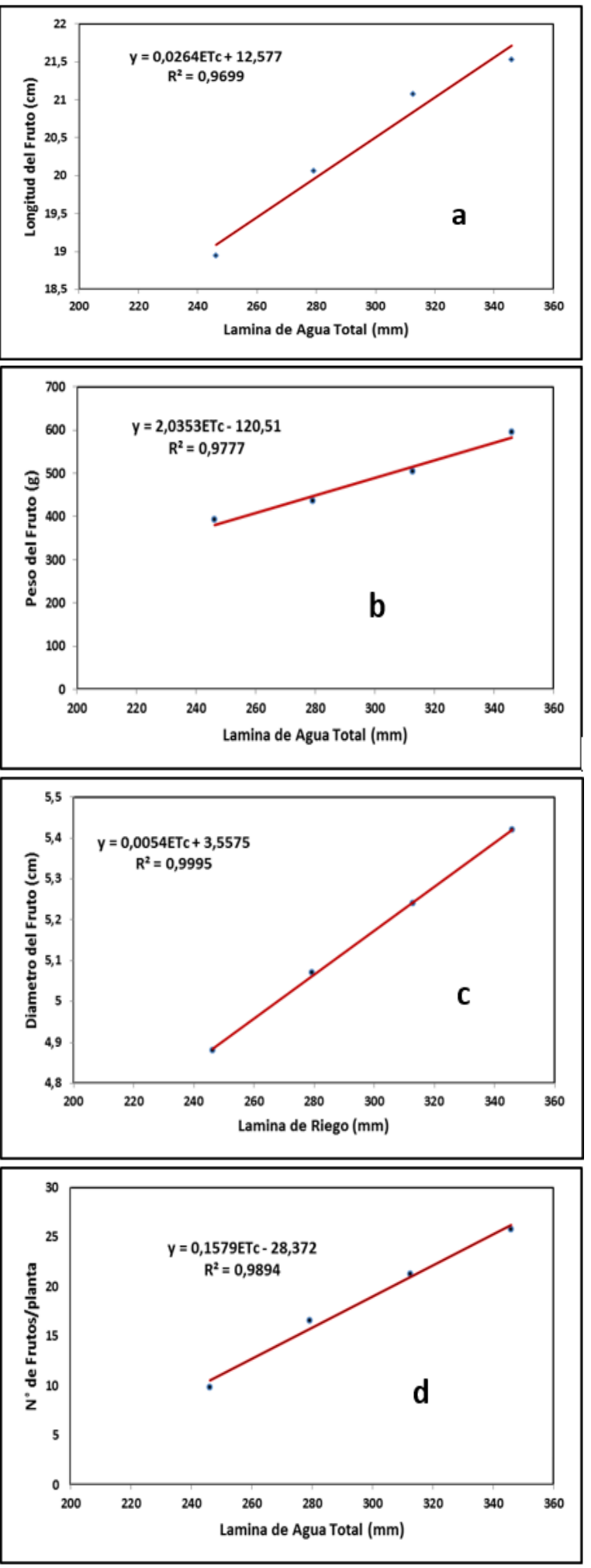

Figura 5...Relación de la lámina de riego aplicada con las variables de calidad (a) Longitud del fruto; (b) peso del fruto; (c) diámetro del fruto y (d) frutos/planta. 


\section{Factor de respuesta de rendimiento $(\mathrm{Ky})$}

La relación lineal entre la disminución proporcional en el consumo de agua y la disminución proporcional en la productividad del rendimiento se representa como el factor de respuesta de la productividad del rendimiento del cultivo (ky); que representa la respuesta de la productividad del rendimiento que se reducirá en el consumo de agua. En otras palabras, explica la disminución de la productividad del rendimiento en relación con la disminución del consumo de agua por unidad (Stewart et al., 1975; Doorenbos y Kassam, 1979). Para su ciclo completo de crecimiento, el factor de respuesta de productividad de rendimiento estacional (ky) se calculó como 2,35 (Figura. 6).

Los valores de ky disminuyen en paralelo con los aumentos de la cantidad de agua (Tabla 3). El valor especificado de ky (2,3487), que es superior a 1,00, indica que la calabacín es altamente sensible al déficit hídrico, con una disminución del rendimiento proporcionalmente mayor que la disminución del uso del agua debido al estrés.

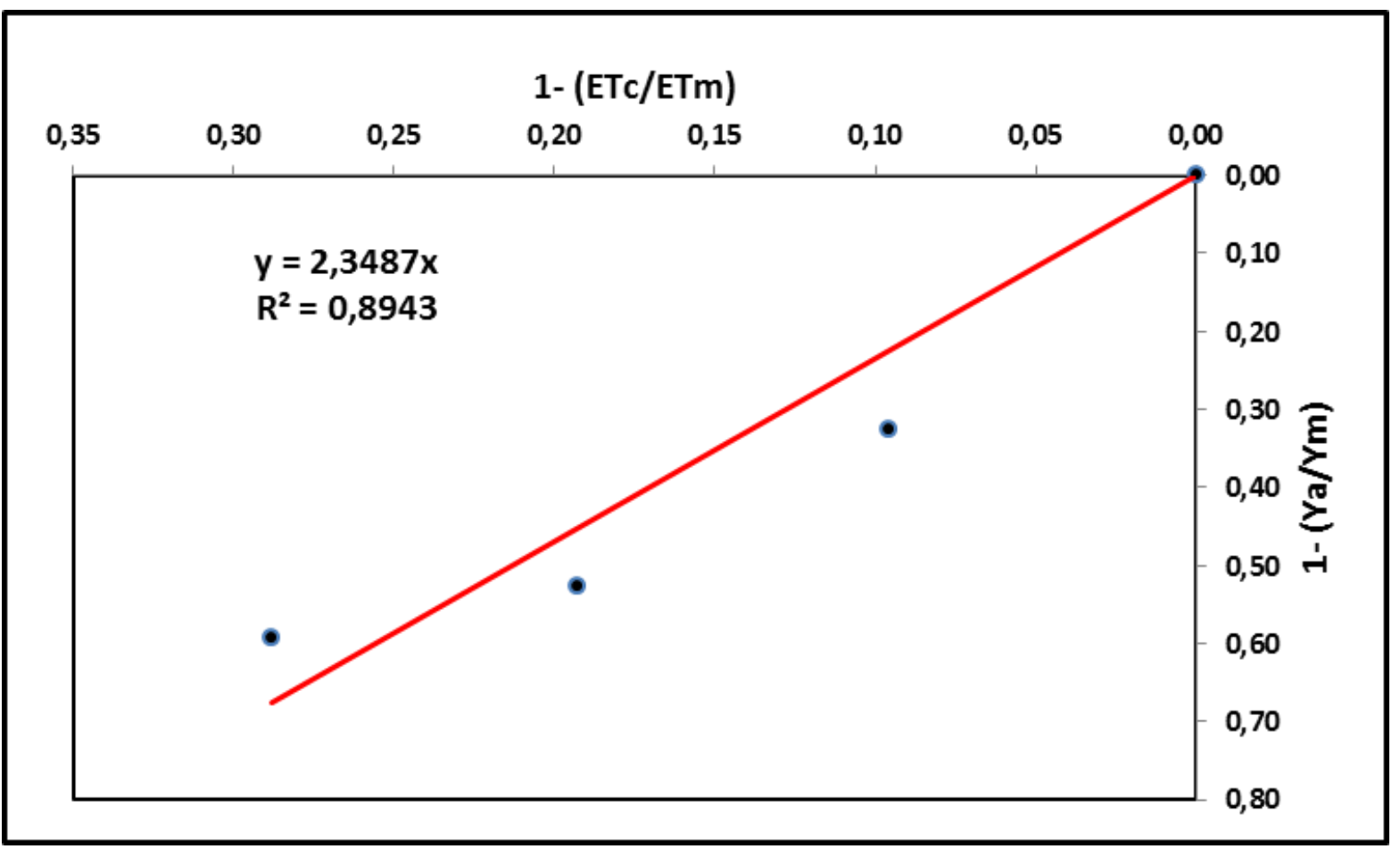

Figura 6. Relación entre la disminución relativa del rendimiento y la disminución relativa de la Evapotranspiración del cultivo en berenjena en su ciclo total de crecimiento.

Tabla 3. Relación de disminución relativa del rendimiento, disminución relativa del uso del agua y factor de respuesta del cultivo para los diferentes tratamientos de riego.

\begin{tabular}{ccccccccc}
\hline $\begin{array}{c}\text { Tratamiento } \\
\text { de Riego }\end{array}$ & $\begin{array}{c}\text { Nivel de Etc } \\
(\%)\end{array}$ & $\begin{array}{c}\text { ETc } \\
(\mathrm{mm})\end{array}$ & $\begin{array}{c}\text { Rendimiento } \\
\text { t/ha }\end{array}$ & ETc/ETm & Ya/Ym & 1-(ETc/ETm) & 1-(Ya/Ym) & Ky \\
\hline T1 & $\mathbf{1 2 0 , 0 0}$ & $\mathbf{3 4 5 , 8 8}$ & 33,39 & 1,00 & 1,00 & 0,00 & 0,00 & 0,00 \\
T2 & 100,00 & 312,68 & 22,49 & 0,90 & 0,67 & 0,10 & 0,33 & 3,40 \\
T3 & 80,00 & 279,28 & 15,83 & 0,81 & 0,47 & 0,19 & 0,53 & 2,73 \\
T4 & 60,00 & 246,28 & 13,57 & 0,71 & 0,41 & 0,29 & 0,59 & 2,06 \\
\hline
\end{tabular}


Yavuz et al. (2015) reporta para los años 2013 y 2014, en el rendimiento relativo de semilla valores de Ky 0,92 y 1,27 respectivamente. Cuando se combinan los valores para ambos años, el valor Ky pasa a 1,08 con un coeficiente de determinación $\left(\mathrm{R}^{2}\right)$ de 0,92 , y una relación estadísticamente significativa a un nivel de $\mathrm{P}$ $<0,01$.Por otro lado, Amer (2011), señala que los rendimientos de frutos y semillas de calabaza se vieron significativamente afectados en una relación lineal $\left(\mathrm{R}^{2}\right.$ $\geq 0,91)$ por las cantidades de riego deficitarias o excedentes aplicadas en el riego; y el factor de respuesta del rendimiento fue de 0,77. Por último, Ertek et al. (2004) encontraron una relación lineal positiva significativa entre el agua de riego, el consumo de agua de la planta, las características de la fruta y el rendimiento.

\section{CONCLUSIONES}

Los objetivos finales de las estrategias óptimas de la gestión de riego en áreas deficitarias son mejorar el rendimiento, la calidad del fruto tanto como sea posible, aumentar la EUA y reducir el consumo de agua. El tratamiento $120 \%$ ETc permitió el máximo rendimiento y calidad (en términos de frutos/planta, longitud, diámetro y peso de la fruta), y la mayor EUA. El máximo rendimiento obtenido fue de $33,39 \mathrm{t} \mathrm{ha}^{-1} \mathrm{y}$ la mayor eficiencia del uso del agua $9,65 \mathrm{~kg} \mathrm{~m}^{-3}$.Todas las funciones de producción del rendimiento y de los parámetros de calidad del fruto obedecen a una ecuación matemática del tipo lineal con un alto valor de coeficiente de determinación $\mathrm{R}^{2}$ por encima del 90 $\%$.El factor de respuesta del rendimiento del cultivo de calabacín $(2,35)$ mayor de 1,00 indica que este cultivo es muy susceptible al déficit de agua. Para la zona de estudio se recomienda una dosis de riego de $120 \%$ de la ETc, la cual es equivalente a una dosis diaria promedio de $4 \mathrm{~mm} /$ día o 1,32 litros/día/planta en la época de floración y formación del fruto de calabacín.

\section{Conflicto de Intereses}

Los autores del presente trabajo de investigación declaran no tener conflicto de intereses.

\section{Contribución de los Autores}

Todos los autores participaron en la conceptualización, metodología, investigación, redacción del manuscrito inicial, revisión bibliográfica, y en la revisión y probación del manuscrito final.

\section{REFERENCIAS}

- Adams, P. (2002). Nutritional control in hydroponics. In: Savvas, D., Passam, H.C.(Eds.), Hydroponic Production of Vegetables and Ornamentals. Embryo Publications, Athens, Greece, pp. 211-261.

- Allen, R. G., Pereira, L. S., Raes, D., \& Smith, M. (1998). Crop Evapotranspiration: Guidelines for Computing Crop Requirements: Irrig. Drain. Paper No. 56. FAO, Rome, Italy.

- Al-Omran, A. M., Sheta, A.S., Falatah, A. M., \& Al-Harbi, A. R. (2005). Effect of drip irrigation on squash (Cucurbita pepo) yield and water-use efficiency in sandy calcareous soils amended with clay deposits. Agric. Water Manage (73), 43-55.

- Amer, K. H. (2011). Effect of irrigation method and quantity on squash yield and quality. Agric. Water Manage. (98), 1197-1206.

- Anwar, M. R., Liu, D. L., Macadam, I., \& Kelly, G. (2013). Adapting agriculture to climate change: a review. Theor. Appl. Climatol. (113), 225-245.

- Bekele, S., \& Tilahum, K. (2007). Regulated deficit irrigation scheduling of onion in a semi arid region of Ethiopia. Agric. Water Manage (89), 148-152.

- Bloch, D., Hoffmann, C. M., \& Marlander, B. (2006). Impact of water supply on photosynthesis, water use and carbon isotope discrimination of sugar beet genotypes. Eur. J. Agron. (24), 218-225.

- Diaz-Perez, J. C., \& Eaton, T. E. (2015). Eggplant (Solanum melongena L.) plant growth and fruit yield as affected by drip irrigation rate. Hortscience .50(11), 1709-1714.

- Doorenbos, J.; \& Kassam, A. H. (1979). Yield response to water. FAO Irrigation and drainage. Paper $\mathrm{N}^{\circ} 33$.

- El-Dewiny, C.Y. (2011). Water and fertilizer use efficiency by squash grown under stress on sandy soil treated with acrylamide hydrogels. J. Appl. Sci. Res. (7),1828-1833.

- El-Gindy, A. G. M., El-Banna, E. S., El-Adl, M.A., \& Metwally, M. F. (2009). Effect of fertilization and irrigation water levels on summer squash yield 
under drip irrigation. Misr Journal of Agricultural Engineering. (26), 94-106.

- El-Mageed, T. A. A., \& Semida, W. M. (2015). Effect of deficit irrigation and growing seasons on plant water status, fruit yield and water use efficiency of squash under saline soil. Scientia Horticulturae. (186), 89-100.

- Ertek, A., Sensoy, S., Kucukyumuk, C., \& Gedik, I. (2004). Irrigation frequency and amount affect yield components of summer squash (Cucurbita pepo L.). Agric. Water Manage. (67), 63-76.

- Ertek, A., Sensoy, S., Gedik, I., \& Kücükyumuk, C. (2006). Irrigation scheduling based on pan evaporation values for cucumber (Cucumis sativus L.) grown under field conditions. Agric. Water Manage (81),159-172.

- FAO [Food and Agriculture Organization]. (2020). Superficie, producción y rendimiento de calabazas en el mundo. www.FAO. org. (Consulta: noviembre 5, 2020).

- Fernández, I., Lecina, S., Ruiz-Sánchez, M.C., Vera, J., Conejero, W., Conesa, M.R., Domínguez, A., Pardo, J.J., Léllis, B.C., \& Montesinos, P. (2020). Trends and Challenges in Irrigation Scheduling in the Semi-Arid Area of Spain. Water 12 (3): 785.

- Fereres, E., \& Soriano, M.A. (2007). Deficit irrigation for reducing agricultural water use. Special issue on Integrated approaches to sustain and improve plant production under drought stress. J. Exp. Bot. (58), 147-159.

- Geerts, S., \& Raes, D. (2009). Deficit irrigation as an on-farm strategy to maximize crop water productivity in dry areas. Agric. Water Manage. (96), 1275-1284.

- Gil, J. A., Montaño, N., \& Plaza, R. (2012). Efecto del riego y la cobertura del suelo sobre la productividad de dos cultivares de ají dulce. Revista Bioagro 24 (2), 43-148.

- Gil, J. A., Montaño, N., \& Valderrama, J. A. (2014). Efecto de cuatro láminas de riego y dos edades de trasplante sobre el rendimiento y producción de dos cultivares de melón (Cucumis melo L.). Revista UDO-Agrícola. 14 (1), 11-21.

- He-xi, Z., Dao-cai, C., Qun, W., Jun, F., \& Xiaoyu, F. (2011). Yield and quality response of cucumber to irrigation and nitrogen fertilization under subsurface drip irrigation in solar greenhouse. Agric. Sci. China. (10), 921-930.

- Hamed, M. F. (1988). Effect of Some Agricultural Practices on Growth and Yield of Rape Plants. Fac. Agric., Ain Shams Univ., Cairo, Egypt (MSc. Thesis).

- Howell, T. A., Cuenca, R. H., \& Solomon, K. H. (1990). Crop yield response. In: G. J. Hoffman, R.A. Howell and K. H. Solomon. (Eds). Management of Farm Irrigation System. ASAE Monograph, St. Joseph, Michigan, United States of America. p. 93-122.

- Igbadun, H. E., Ramalan, A. A., \& Oiganji, E. (2012). Effects of regulated deficit irrigation and mulch on yield, water use and crop water productivity of onion in Samaru, Nigeria. Agric. Water Manage. (109), 162-169.

- Igbadun, H. E., Salim, B. A., Tarimo, A. K. P. R., \& Mahoo, H. F. (2008). Effects of deficit irrigation scheduling on yields and soil water balance of irrigated maize. Irrig.Sci. (27), 11-23.

- Jones, H.G. (2004). What is water use efficiency? In: Bacon, M.A. (Ed.), Water Use Efficiency in Plant Biology. Blackwell Publishing, Oxford, UK, pp. 27-41.

- Karam, F., Lahoud, R., Masaad, R., Daccache, A., Mounzer, O., \& Rouphael, Y. (2006). Water use and lint yield response of drip irrigated cotton to the length of irrigation season. Agric. Water Manage. (85), 287-295.

- Kirda, C. (2002). In: FAO Corp. Doc. Rep. (Ed.), Deficit Irrigation Scheduling Based on Plant Growth Stages Showing Water Stress Tolerance. Deficit Irrigation Practices, vol. 22. FAO Corp. Doc. Rep., Rome, pp. 3-10.

- Malash, N., Flowers, T. J., \& Ragab, R. (2005). Effect of irrigation systems and water management practices using saline and non-saline water on tomato production. Agric. Water Manage. (78), 25-38.

- Montaño, N.J., Gil Marín, J.A., \& Yeniledys, P. (2018). Rendimiento de pepino (Cucumis sativus L.) en función del tipo de bandeja y la edad de trasplante de las plántulas. Anales Científicos .79 (2), 377 - 385.

- Okasha, E., Fad, M., Hashem, A., \& El-Metwally I. M. (2020). Effect of Irrigation System And Irrigation Intervals On The Water Application 
Efficiency, Growth, Yield, Water Productivity And Quality Of Squash Under Clay Soil Conditions. Plant Archives. 20(2): 3266-3275.

- Ozbahce, A.; \& Tari, A. F. (2010). Effects of different emitter space and water stress on yield and quality of processing tomato under semi-arid climate conditions. Agric.Water Manage. (97), 1405-1410.

- Passioura, J. B.; \& Angus, J. F. (2010). Improving productivity of crops in water-limited environments. - In: Sparks, D. L. (ed.). Advances in Agronomy. (106), 37-75.

- Pereira, L. S., Oweis, T., \& Zairi, A. (2002). Irrigation management under water scarcity. Agric. Water Manage. (57), 175-206.

- Rolbiecki, R., Rolbiecki, S., Podsiadło. C., Wichrowska.D, Figas, A., Jagosz, B, \& Wiesław Ptach. (2017). Influence of Drip Irrigation On the Yielding Of Summer Squash 'White Bush' Under Rainfall thermal Conditions Of Bydgoszcz And Stargard. $\mathrm{Nr}$ Iii/2/2017, Polish Academy of Sciences, Cracow Branch, pp. 1229-1240.

- Rouphael, Y.; \& Colla, G. (2005). Growth, yield, fruit quality and nutrient uptake of hydroponically cultivated zucchini squash as affected by irrigation systems and growing seasons. Sci. Hortic. (105), 177-195.

- Scholberg, J., McNeal, B. L., Jones, J. W., Boote, K.J., Stanley, C.D., \& Obreza, T.A. (2000). Growth and canopy characteristics of field-grown tomato. Agron. J. (92), 152-159.

- Solomon, K. H. (1985). Tropical crop water production functions. Winter Meeting. ASCE, Chicago, Illinois, United State of America. (85), 17-20.

- Stewart, J. I., Misra, R.D., Pruitt, W.O., \& Hagan, R.M. (1975). Irrigating corn and sorghum with a deficient water supply. Trans. ASAE. (18), 270280.

- Wajid, S. A. (1990). Effect of Different Mulching Material and Irrigation Levels on Growth and Grain Yield of Maize. Dept. Agron. Univ. Agric., Faisalabad (M Sc.Agric. Thesis).

- Wang, Z. Y., Liu, Z.X., Zhang, Z.X., Zhang, Z.K., \& Liu, X. B. (2009). Subsurface drip irrigation scheduling for cucumber (Cucumis sativus L.) grown in solar greenhouse based on $20 \mathrm{~cm}$ standard pan evaporation in Northeast China. Sci. Hortic. (123), 51-57.

- Wetzel, J., \& Stone, A. (2019). Yield Response of Winter Squash to Irrigation Regime and Planting Density. HORTSCIENCE. 54 (7), 1190-1198.

- Yavuza, D., Seymen, M., Yavuz, N., \& Türkmen O. (2015). Effects of irrigation interval and quantity on the yield and quality of confectionary pumpkin grown under field conditions. Agricultural Water Management. (159), 290-298. 